\title{
Dysarthrie et troubles de la déglutition dans la myosite à inclusions Revue de la littérature sur les méthodes d'évaluation et les prises en charge orthophoniques
}

La myosite à inclusions est une pathologie neuromusculaire rare touchant principalement les adultes âgés de plus de 50 ans. L'incidence de la dysphagie y est la plus élevée au sein des myopathies inflammatoires. La dysarthrie est un symptôme clinique pouvant également être présent. Actuellement, aucune revue ne s'est spécifiquement intéressée à la place occupée par l'orthophoniste dans l'évaluation et la prise en charge de cette pathologie. Cette revue de la littérature a pour but de faire un état des lieux de la recherche scientifique concernant l'évaluation de la dysarthrie et de la dysphagie dans la myosite à inclusions ainsi que des méthodes de rééducation orthophonique existantes.

Clara Solman

Université de Lille,

Département

d'Orthophonie Gabriel

Decroix, Lille, France

Vincent Sobanski

Univ. Lille, U995, Lille

Inflammation Research

International Center

(LIRIC), CHU Lille,

département de médecine

interne et immunologie

clinique F-59000 Lille,

France

Sylvie Nguyen

Université de Lille, CHU

de Lille, Centre de

référence des maladies

neuromusculaires

Nord-Est-Île-de-France,

Lille, France

Céline Tard

Université de Lille,

U1171, CHU de Lille,

Centre de référence des

maladies

neuromusculaires

Nord-Est-Île-de-France,

Service de neurologie,

Lille, France

Contact

celine.tard@chru-lille.fr

\section{Introduction}

La myosite à inclusions est une myopathie chronique et progressive caractérisée par une faiblesse musculaire touchant certains groupes musculaires spécifiques. Il s'agit de la myopathie inflammatoire la plus fréquemment retrouvée chez les personnes de plus de 50 ans, devenant ainsi la myopathie la plus souvent associée au vieillissement.

La dysphagie est une des manifestations cliniques de la myosite à inclusions et peut même en être un symptôme de présentation [1]. Cette dernière pouvant se révéler sévère, elle a un impact important sur le pronostic et la qualité de vie de ces patients. La dysarthrie est également un symptôme relativement présent dans la clinique des myosites. On peut ainsi observer lors de l'examen clinique une faiblesse linguale, des cordes vocales flasques ainsi que des mouvements palataux de faible amplitude [2].

L'objectif de cette revue de la littérature vise à faire un état des lieux de la recherche scientifique sur le thème de la dysphagie et de la dysarthrie dans la myosite à inclusions en s'interrogeant sur les critères d'évaluation.

Cette pathologie ayant été peu étudiée au niveau orthophonique, le second objectif de cette revue de la littérature est de combler le manque de ressources à ce sujet en étudiant les méthodes de rééducation orthophonique proposées.

\section{Méthode}

Cette revue de la littérature est uniquement composée d'articles issus de la base de données Pubmed en raison de leur validité scientifique.

Le protocole dont nous nous sommes inspirés pour la réalisation de cette revue de la littérature est tiré du "Preferred Reporting Items for Systematic review and Meta-Analyses " d'après la traduction française des lignes directrices PRISMA [3]. La première étape de la création de cette revue a consisté en la recherche de mots-clés MeSH. L'équation finale choisie permettant de regrouper le maximum d'articles sur le sujet a été "Inclusion body myositis" AND "dysphagia". Les publications étant peu nombreuses pour la dysarthrie, l'association de mots-clés choisie a été "Inclusion body myositis" AND "dysarthria " OR " speech ». Deux articles ont été proposés par la base de données mais ces derniers n'ont pas pu être exploités.

Compte tenu du faible nombre de parutions concernant notre sujet, les articles et les résumés potentiellement éligibles n'ont pas été restreints quant à leurs dates de publication. De même, une structure IMRAD n'a pas été définie comme nécessaire à l'éligibilité de l'article. Le recueil des articles sur Pubmed s'est effectué de janvier 2016 à novembre 2017. Des critères d'inclusions ont été définis : 
- Sujet principal : "Inclusion Body Myositis" "dysphagia/dysarthria".

- Présence des termes: "evaluation", "swallowing", "swallowing rehabilitation", "speech therapist".

- Langues : français, anglais.

- Les doublons ont été exclus.

Après sélection des articles, nous avons procédé à un classement des articles suivant leur année de publication, le type d'étude et les méthodes d'évaluation utilisées. Le même procédé a été appliqué concernant les préconisations de prise en charge orthophonique. Les éléments obtenus répondant à notre problématique ont ensuite été triés puis rassemblés sous forme de tableaux. Les articles y sont répertoriés selon leur date de parution, de la plus récente à la plus ancienne.

\section{Résultats}

Les différentes phases du traitement des articles, à savoir leur identification, leur éligibilité et leur inclusion à la revue, sont présentées dans la figure 1 . Nos deux associations finales nous ont permis de recenser sur Pubmed 81 articles. Vingt-trois articles ont été sélectionnés pour être intégrés dans la revue de littérature finale.

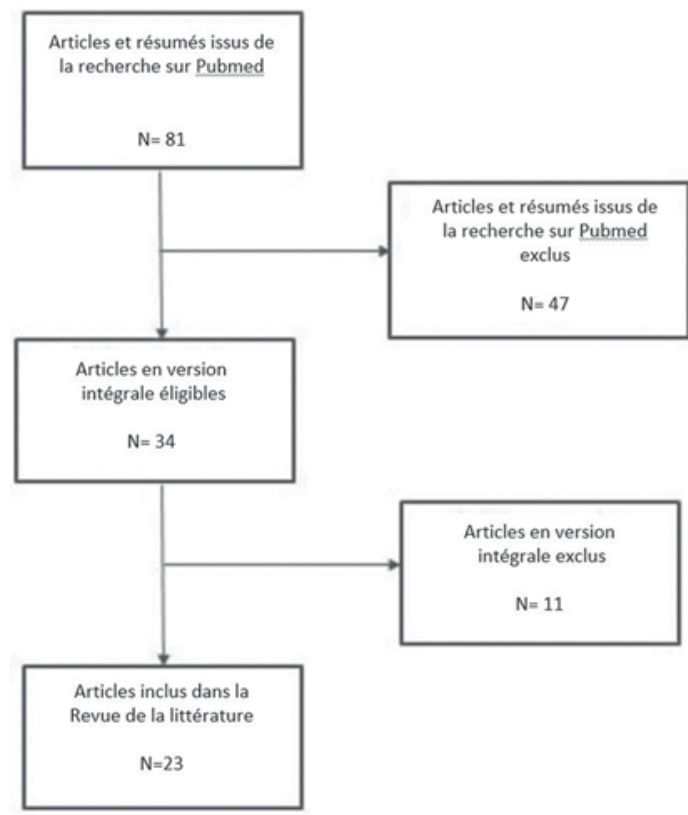

Figure 1

Diagramme de flux ( $\mathrm{N}$ : nombre d'articles).

Méthodes d'évaluation

Les méthodes d'évaluation regroupent les questionnaires auto-administrés et les techniques d'imagerie. Dans les études associant questionnaire et technique d'imagerie, le questionnaire permettait de récolter la présence d'une plainte et d'ainsi d'objectiver à l'imagerie le retentissement fonctionnel du trouble décrit. Un examen clinique est quelquefois compris dans le protocole d'évaluation de l'étude de la dysphagie. Pour la plupart, les questionnaires sont administrés lors de consultations ou durant un entretien téléphonique. Les questionnaires proposés par téléphone peuvent être administrés en première intention ou à distance des évaluations. Sur les vingttrois articles composant la revue de littérature, six contiennent un questionnaire. Les questions posées appartiennent pour la plupart à des questionnaires validés et reconnus comme le Deglutition Handicap Index [4], le Swallowing Related Quality of Life Questionnaire [5], le Sydney Swallow Questionnaire [6] ainsi que le MD Anderson Dysphagia Inventory [7].

L'examen clinique n'est pas systématiquement proposé lors des expérimentations. En effet, il apparaît au sein de quatre articles sur les vingt-trois présentés.

Des explorations invasives sont présentes dans chaque article et regroupent plusieurs techniques. La vidéofluoroscopie est la technique d'imagerie médicale la plus répandue dans l'évaluation de la dysphagie. En effet, on la retrouve dans dix-neuf des vingt-trois articles. Elle peut être complétée par d'autres méthodes d'imagerie mais reste l'unique examen pratique au sein de sept articles. La manométrie oesophagienne est souvent proposée en complément de la vidéofluoroscopie. Leur association est retrouvée dans sept articles.

Préconisations d'interventions orthophoniques Les adaptations de textures alimentaires concernent dans un premier temps l'exclusion des aliments problématiques, à savoir ceux dont la texture est caoutchouteuse, à double texture ou dont les éléments se dispersent. Ensuite, la texture est adaptée en fonction des difficultés avec une proposition d'aliments semi-solides et/ou de liquides épaissis.

Les techniques de compensation comprennent des adaptations posturales telles que l'anté-flexion du cou et la rotation du cou mais aussi de nombreuses adaptations en fonction du déficit observé. La "déglutition d'effort " permet d'augmenter la propulsion du bol alimentaire et d'améliorer la contraction du pharynx tandis que la déglutition supra-glottique permet une fermeture précoce et volontaire des cordes vocales.

La manouuve de Mendelsohn est citée dans plusieurs articles, son but est de favoriser l'élévation laryngée ainsi que l'ouverture du sphincter supérieur de l'œesophage. En effet, la dysphagie touchant les 
personnes atteintes de myosite à inclusions est caractérisée par un dysfonctionnement du sphincter supérieur de l'œesophage. Les fausses routes secondaires sont prévenues grâce aux raclements de la gorge, aux doubles déglutitions et à l'utilisation de la toux volontaire après chaque déglutition.

Des exercices de déglutition ont également été proposés à certains patients afin d'être réalisés en plus des temps de repas. Certaines techniques de compensation sont réutilisées sous forme d'exercices dans un but de renforcement et d'automatisation. Nous pouvons citer la déglutition d'effort, la déglutition supra-glottique mais également la manœuvre de Mendelsohn.

\section{Discussion}

La problématique de la dysphagie au sein de la myosite à inclusions est particulièrement relayée par des études de cas, pour la plupart transversales. Le manque de données longitudinales peut se justifier par la rareté de cette pathologie. Les recherches existantes comprennent des études de type cohorte ou étude de cas. Les études de cohorte, à plus grande échelle, sont peu nombreuses et souvent rétrospectives. Les études de cas posent quant à elles la question de la représentativité de l'étude et de la généralisation des résultats ou observations obtenus à l'ensemble de la population. Globalement, les articles traitant de cette problématique se situent entre un niveau 3 et un niveau 4 de preuves. Ces derniers correspondant à un faible niveau de preuves scientifiques d'après les recommandations de la Haute Autorité de Santé.

\section{L'examen clinique}

La plupart des études sur la dysphagie ont recours à l'utilisation d'examens d'imagerie, parfois en première intention, sans avoir fait état d'un entretien préalable ou d'un premier examen clinique de la dysphagie. Dans le cadre de la myosite à inclusions, cet examen clinique a pu être réalisé à partir d'observations cliniques simples comme le "test au verre d'eau " même s'il n'est pas explicitement cité sous cette appellation. Ces tests consistent en la déglutition de liquides et/ou de solides de type biscuit sec sous l'observation d'un orthophoniste, d'un médecin ou d'un ergothérapeute [8]. Une échelle de prise alimentaire orale a également été proposée [9]. La FOIS (Functional Oral Intake Scale) est une échelle de 7 points conçue pour désigner le type de texture et la quantité pouvant être gérés en toute sécurité par le patient.

Cependant, l'examen clinique est pratiqué par un orthophoniste dans seulement trois articles. L'examen clinique semble ainsi être très peu exploité dans la littérature scientifique et aucun protocole de référence n'est utilisé. Les indications le concernant restent très succinctes et ne permettent en aucun cas sa reproductibilité.

\section{Les questionnaires}

Parmi les procédés non invasifs, les questionnaires permettent d'interroger le patient selon des points précis, standardisés, et de récolter des informations ciblées et également standardisées. Les questionnaires sont reproductibles et peu onéreux. C'est une méthode non invasive et rapide qui ne présente que très peu de contraintes. La plupart des études l'employant attestent de son importance pour une utilisation en première ligne, dans un contexte d'entretien avec le patient et précédant l'imagerie médicale [9, 10]. Pour autant, le questionnaire n'est pas systématiquement utilisé.

Par ailleurs, les questionnaires cités et utilisés pour évaluer la dysphagie ne sont pas tous standardisés et validés. Actuellement, aucun n'est spécifique aux troubles de la déglutition au sein des myopathies inflammatoires. Nous pouvons toutefois relever que le Sydney Swallowing Questionnaire [6] semble être le questionnaire le plus spécifique car il est dédié à l'évaluation de la déglutition chez les patients atteints de dysphagie oropharyngée d'origine neuromyogénique.

Le questionnaire présente un intérêt non négligeable compte tenu du nombre d'études ayant montré que les patient atteints de myosite à inclusions étaient les plus touchés par la dysphagie par rapport aux autres myopathies inflammatoires telles que la polymyosite et la dermomyosite [1, 9]. De plus, peu de patients rapportent spontanément à leur praticien leurs difficultés pour s'alimenter et l'utilisation d'un questionnaire ou de quelques questions ciblées permettent de mettre en avant un potentiel trouble de la déglutition et d'agir ainsi en amont de l'installation des troubles.

La dysphagie pouvant également être un des symptômes de présentation de la myosite à inclusions durant plusieurs années, certains auteurs préconisent d'inclure des questions portant sur la dysphagie à la batterie d'évaluation classique administrée aux patients présentant des myopathies inflammatoires [11]. Les questions les plus sensibles à la détection d'une dysphagie sont : "la nourriture se coince-t-elle dans votre gorge?", "avez-vous à avaler à plusieurs reprises pour avaler toute la nourriture?", "est-ce que vous toussez durant les repas?" et "avez-vous des difficultés pour avaler la nourriture solide?" et peuvent permettre d'évoquer la présence d'une potentielle dysphagie, objectivable lors d'une vidéofluoroscopie [9]. 
Les méthodes d'exploration fonctionnelles

Les techniques utilisées comprennent le gold-standard des évaluations de la déglutition, à savoir la vidéofluoroscopie, couplée à la manométrie œesophagienne ou à l'endoscopie. Pour autant, aucun protocole n'est actuellement établi et repris par les auteurs. Il est à noter qu'il y a une absence de critères d'observation de la dysphagie pré-établis car peu d'études emploient des échelles d'observation lors de leur examen vidéofluoroscopique. Certains articles ne mentionnent aucun élément technique pouvant permettre de reproduire des conditions d'évaluation identiques. Ce manque d'exhaustivité se retrouve notamment dans les études plus anciennes. Une seule étude mentionne la présence de deux orthophonistes experts ayant assisté à la vidéofluoroscopie sur trois patients. Les résultats avaient montré un excellent effet inter-juges [9].

L'association de la manométrie œesophagienne et de la vidéofluoroscopie est recommandée afin d'étudier la préservation de la force du sphincter pharyngoœesophagien et d'apprécier l'efficacité prévisible de techniques chirurgicales envisagées [12].

En comparaison à d'autres pathologies plus connues et étudiées, ce manque de standardisation dans les études traitant d'une même symptomatologie pourrait s'expliquer en raison du faible nombre d'études encore parues sur cette problématique. L'origine de la dysphagie dans la myosite à inclusions peut être due à un défaut d'ouverture du sphincter supérieur de l'œesophage et d'une faiblesse musculaire affectant le muscle crico-pharyngé. L'imagerie médicale permet d'objectiver la physiopathologie des troubles observés.

Les approches rééducatives orthophoniques

Peu d'études font référence aux méthodes de rééducation orthophoniques pouvant être proposées aux patients atteints de myosite à inclusions. Actuellement, il n'existe aucune recommandation spécifique. Les interventions les plus pratiquées visant la réhabilitation de la déglutition sont chirurgicales (myotomie du muscle crico-pharyngé), locales (injection de toxine botulique $\mathrm{A}$ ), ou médicamenteuses avec la prise d'immuno-modulateurs. Ainsi, peu d'études s'intéressent à l'effet de l'exercice sur la déglutition et la prise en charge orthophonique ne semble pas faire partie des alternatives principales proposées. Une étude a d'ailleurs souligné le fait qu'aucun des patients atteints de myosite à inclusions n'avait vu

d'orthophoniste malgré la présence de troubles de la déglutition déjà fortement installés [9]. Les patients avaient tendance à adapter eux-mêmes leur façon de s'alimenter, notamment en se concentrant davantage sur leur mastication et en évitant les distractions extérieures.

Woissard-Bassols et Puech [13] ont élaboré des indications de prise en charge orthophonique dans le cadre du dysfonctionnement du sphincter supérieur de l'œesophage. Concernant les stratégies d'adaptation, le bol alimentaire doit avoir de préférence une consistance davantage liquide que solide avec des prises en petites quantités. Durant le repas, la flexion antérieure est préconisée. Les principales manœuvres recommandées sont les déglutitions répétées à vide, l'alternance de liquides et de solides durant les repas, l'utilisation de la manœuvre de Mendelsohn ainsi que la stimulation externe du plancher buccal. La rééducation spécifique se scinde en exercices analytiques et exercices fonctionnels. La rééducation analytique est basée sur un travail de la musculature laryngée extrinsèque, le protocole cervical de Shaker (mouvements de flexion de tête quotidien d'une durée de 6 semaines afin de muscler les élévateurs du larynx) et le travail pharyngé. La rééducation fonctionnelle passe quant à elle par la réalisation de la manœeuvre de Mendelsohn et de la déglutition tête en extension.

\section{La manoeuvre de Mendelsohn}

Le seul élément de rééducation propre à la myosite à inclusions semble être la manœuvre de Mendelsohn car elle est spécifique à l'ouverture du sphincter supérieur de l'œesophage, déficiente dans cette pathologie. Cette technique consiste à prolonger volontairement l'élévation laryngée de quelques secondes afin de favoriser une meilleure et plus longue ouverture du sphincter supérieur de l'œesophage durant la déglutition. Pour effectuer cette manœeuvre, on demande au patient d'avaler en se concentrant sur l'ascension du larynx, de le maintenir en position haute durant quelques secondes après la fin de la déglutition puis de le relâcher. L'accompagnement peut être réalisé manuellement, prodigué par le patient lui-même ou un thérapeute (le plus souvent orthophoniste). Il est également possible de maintenir volontairement le larynx en position haute sans soutien manuel, grâce à de l'entraînement et en se concentrant sur l'ascension de ce dernier durant la déglutition.

Même si d'un point de vue physiopathologique, cette manœuure semble être relativement adaptée à la problématique d'ouverture du sphincter supérieur de l'œsophage, elle est pourtant limitée du fait de l'atteinte neuromusculaire. En effet, peu d'études ont pu évaluer cette manœuuve dans le cadre de myopathies inflammatoires. La majorité d'entre elles sont rétrospectives et intriquées avec d'autres recommandations et adaptations, elles ne permettent pas 
d'observer une évolution due à l'utilisation spécifique de cette méthode. De plus, cette pathologie étant peu commune, le manque d'expertise clinique de l'orthophoniste d'une part et les capacités restreintes du patient d'autre part peuvent également être un frein à l'utilisation de cette manœuvre [1].

\section{La dysarthrie}

Aucune étude ne s'est penchée sur la question de la dysarthrie dans la myosite à inclusions. Pourtant, la voix dans les myopathies inflammatoires est qualifiée de soufflée, de nasale et d'enrouée [14]. Les examens moteurs mettent en avant une faiblesse linguale, un manque de tonicité des cordes vocales et une altération des mouvements vélaires [2]. Néanmoins, aucune étude de notre sélection n'y a fait spécifiquement référence. Comme évoquée lors de nos hypothèses, la raison de ce manque de données peut être due au caractère moins alarmant et prioritaire de la dysarthrie, survenant dans un contexte de pathologie rare. Cette dernière est donc peu étudiée. Les préconisations spécifiquement recommandées pour la myosite à inclusions étant peu nombreuses concernant la dysphagie, celles pour la dysarthrie sont pour le moment inexistantes. Généralement, sa prise en charge est effectuée en première intention par l'orthophoniste. Nous pouvons ainsi estimer qu'il en est de même lorsqu'elle survient dans un contexte de myosite à inclusions.

\section{Conclusion}

La myosite à inclusions est une pathologie rare, encore peu étudiée au regard d'autres maladies neuromusculaires inflammatoires. La dysphagie étant un symptôme très présent et à l'origine de nombreuses complications, plusieurs études tendent à s'y consacrer afin de proposer les meilleures procédures interventionnelles. Les méthodes d'évaluation de la dysphagie dans la myosite à inclusions ne sont pas différentes de celles utilisées dans d'autres pathologies. La vidéofluoroscopie ainsi que la manométrie oesophagienne sont les principaux outils d'investigation. Outre l'imagerie, les questionnaires semblent se répandre peu à peu.

Le rôle de l'orthophoniste concernant l'évaluation de la dysphagie dans le cadre de la myosite à inclusions semble être encore à définir, il n'existe actuellement aucun consensus entre les auteurs.

L'orthophoniste ne fait pas encore partie du protocole d'évaluation de la dysphagie dans la myosite à inclusions mais des études ont montré que son expertise pouvait être requise, notamment lors de l'examen clinique, de l'administration de questionnaires et de l'observation de la vidéofluoroscopie. Actuellement, les protocoles de remédiation sont pour la majorité chirurgicaux ou médicamenteux. Les bénéfices des techniques compensatrices de la déglutition et des exercices spécifiques ne sont pour le moment pas observables. En effet, les études sont majoritairement rétrospectives et ne permettent pas d'observer un réel avantage à l'utilisation de ces méthodes de prise en charge orthophonique.

Des études prospectives devront être entreprises afin de répondre à cette interrogation, notamment concernant la manœuure de Mendelsohn qui pourrait se révéler efficace en raison de son action directe sur l'ouverture du sphincter supérieur de l'œesophage, principalement altérée dans la myosite à inclusions. Malgré son objectivation lors d'examens cliniques, la dysarthrie est un symptôme qui n'a pas été pour le moment spécifiquement étudié.

\section{Summary}

Dysarthria and swallowing disorders in inclusion body myositis. Review of the literature on assessment methods and speech therapy

Background: Inclusion body myositis is a rare neuromuscular disease affecting mainly adults over 50 years. The incidence of dysphagia is highest in inclusion body myositis among inflammatory myopathies. Dysarthria is a clinical symptom that may also be present. Currently, no review has looked at the place occupied by the speech therapist in the evaluation and the management of this pathology.

Objective: This review of the literature aims to make an inventory of scientific research for the evaluation of dysarthria and dysphagia in inclusion body myositis as well as existing speech therapy rehabilitation methods.

Method: With a selection of 81 articles from the Pubmed database, 23were chosen to integrate the final literature review.

Results: The results suggest that speech-language therapists seldom participate in the assessment of dysphagia. The effectiveness of rehabilitation strategies for swallowing is still under discussion, but indications on the rehabilitation are present. Regarding dysarthria, no study has referred to.

Conclusion: The role of the speech-language therapist regarding the assessment of dysphagia in the context of inclusion body myositis seems to be still defined; there is currently no consensus among the authors.

Key words: dysphagia, dysarthira, inclusion body myosistis, evaluation, swallowing rehabilitation, speech therapist.

\section{LIENS D'INTÉRÊT}

Les auteurs déclarent n'avoir aucun lien d'intérêt concernant les données publiées dans cet article. 


\section{RÉFÉRENCES}

1. Oh TH, Brumfield KA, Hoskin TL, Kasperbauer JL, Basford JR. Dysphagia in inclusion body myositis: clinical features, management, and clinical outcome. Am J Phys Med Rehabil 2008; 87 : 883-9.

2. Ebert EC. Review article: the gastrointestinal complications of myositis. Aliment Pharmacol Ther 2010 ; 31 : 359-65.

3. Gedda M. Traduction française des lignes directrices PRISMA pour l'écriture et la lecture des revues systématiques et des métaanalyses. Rev Francoph Int Rech Infirm 2017 ; 3 : 19-24.

4. Woisard V, Andrieux M, Puech M. Validation d'un questionnaire d'auto-évaluation du handicap pour les troubles de la déglutition oropharyngée (deglutition handicap index). Rev Laryngol Otol Rhinol (Bord) 2006 ; 5 : 315-25.

5. McHorney C, Bricker D, Robbins J, Kramer A, Rosenbek J Chignell K. The SWAL-QOL outcomes tool for oropharyngea dysphagia in adults. II. Item reduction and preliminary scaling. Dysphagia $2000 ; 15: 122-33$

6. Wallace KL, Middleton S, Cook IJ. Development and validation of a self-report symptom inventory to assess the severity of oralpharyngeal dysphagia. Gastroenterology 2000 ; 118 : 678-87. 7. Chen A, Frankowski R, Bishop-Leone J, Hebert T, Leyks S, Lewin $\mathrm{J}$, et al. The development and validation of a dysphagiaspecific quality-of-life cancer: the M.D. Anderson dysphagia inventory. Arch Otolaryngol Head Neck Surg 2001 ; 127 : 870-6.
8. Knuijt S, Kalf JG, de Swart BJM, Drost G, Hendricks HT, Geurts ACH, et al. Dysarthria and dysphagia are highly prevalent among various types of neuromuscular diseases. Disabil Rehabil 2014 ; 36 : 1285-9.

9. Mulcahy KP, Langdon PC, Mastaglia F. Dysphagia in inflammatory myopathy: self-report, incidence, and prevalence. Dysphagia 2012; 27 : 64-9.

10. Cox FM, Verschuuren JJ, Verbist BM, Niks EH, Wintzen AR, Badrising UA. Detecting dysphagia in inclusion body myositis. $J$ Neurol 2009 ; 256 : 2009-13.

11. Shibata S, Izumi R, Hara T, Ohshima R, Nakamura N, Suzuki $\mathrm{N}$, et al. Five-year history of dysphagia as a sole initial symptom in inclusion body myositis. J Neurol Sci 2017 ; 381 : 325-7.

12. Murata K, Kouda K, Tajima F, Kondo T. A dysphagia study in patients with sporadic inclusion body myositis (s-IBM). Neurol Sci 2012 ; 33 : 765-70.

13. Woissard-Bassols V, Puech M. La réhabilitation de la déglutition chez l'adulte, le point sur la prise en charge fonctionnelle. $2^{e}$ édition revue et argumentée. Le monde du verbe. Bruxelles De Boeck Supérieur, 2011.

14. Ko EH, Rubin AD. Dysphagia due to inclusion body myositis: case presentation and review of the literature. Ann Otol Rhino Laryngol 20.

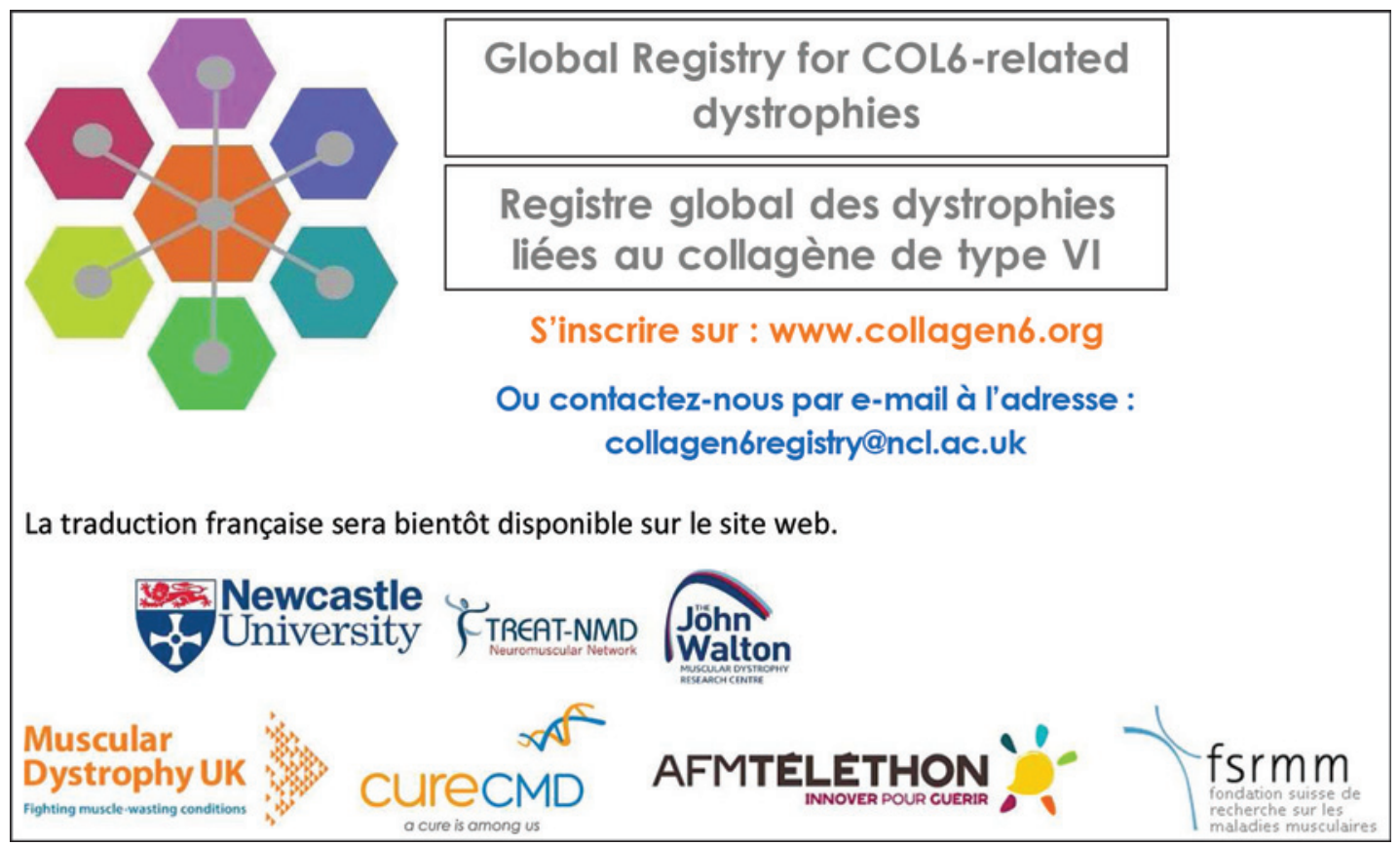

